\title{
Association between shift work and obesity among a group of Iranian military personnel in 2016
}

\author{
Ghanbary Sartang A, MSc ${ }^{1 *}$, Ashnagar M, MSc ${ }^{2}$, Abedi M, MSc ${ }^{3}$, Habibi E, $\mathrm{PhD}^{4}$ \\ 1- MSc in Occupational Health Engineering, School of Health, Isfahan University of Medical Sciences, Isfahan, Iran. \\ 2- MSc in Industrial Psychology, Dept. of Industrial Psychology, Islamic Azad University of Marvdasht, Shiraz, Iran. \\ 3-MSc in Student Health, Safety and Environment (HSE), Center Tehran Azad University of Medical Sciences, Tehran, \\ Iran. 4-Professor, Dept. of Occupational Health Engineering, School of Health, Isfahan University of Medical Sciences, \\ Isfahan, Iran.
}

\begin{abstract}
Received: October 2017, Accepted: October 2017

Background: Nowadays, shift work is a common work schedule. Shift work has been hypothesized as a risk factor for obesity. So, identifying the effective factors on obesity is of great importance. In the present research, we are going to study the effects of shift work on obesity among military personnel in 2016.

Materials and Methods: This descriptive study was carried out on 80 man military at southern Iran in June and July 2016. Respondents were divided into two groups based on their working schedule (40 shift work and 40 day work personnel). These two groups were matched in terms of type work. In this study, the participants were selected through simple random sampling. In this research, we controlled the effect of age and work experience on obesity. Finally, data analysis was performed with SPSS software.

Results: Total people who participated in this study were 80 . The findings revealed that mean body weight and body mass index (BMI) in shift workers were more than day workers. The results also showed that $79.6 \%$ of subjects had BMI $>25$ in shift work individuals. Furthermore, $83.9 \%$ of subjects had BMI $<25$ in day work individuals. Finally, Pearson correlation test showed a significant correlation between BMI $(r=0.69)$ and weight $(r=0.67)$ with years on shift work, in a way that with increasing duration of shift work, BMI and weight increased.

Conclusions: The duration of shift work was positively associated with the prevalence of obesity in military personnel and shall be taken to intervention actions in shift works.
\end{abstract}

Keywords: Obesity, Body Mass Index, Shift Work, Military Personnel

\section{Introduction}

Shift work is a work that takes place on a schedule outside the traditional daily 7 am to $6 \mathrm{pm}$ frame. It can involve evening or night, early morning, and rotating shifts. Many industries rely heavily on shift work, and millions of people work in jobs that require shift schedules. Shift work is the social phenomenon which is an inseparable part of providing services (1).

Obesity is a risk factor for chronic diseases, and a number of studies report thatoverweight and obesity are more prevalent among shift workers than day workers. Obesity is a medical condition in which excess body fat has accumulated to the extent that may have a negative effect on health (2). Obesity is a risk factor for chronic diseases, and a number of studies have reported that overweight and obesity are more prevalent among shift workers more day workers. Shift work-related abnormalities in circulating lipids, central obesity, and hypertension have been repeatedly reported (3). Nowadays, obesity is considered as one of the health problems that increases the risk of some diseases. Overweight and obesity have complex and

* Corresponding author: Ayoub Ghanbary Sartang, School of Health, Isfahan University of Medical Sciences, Isfahan, Iran.

E-mail: aioobghanbary@ymail.com 
multifactorial etiologies and have reached global epidemic proportions. Weight gain is one of the major risk factors for an increase in blood pressure and lipids, and a decrease in glucose tolerance (4). There is growing evidence suggesting that shift work, particularly night shifts, is harmful to workers' health and safety. A research has suggested a relationship between shift work and obesity and an increased risk of suffering from type 2 diabetes (5). Dorrian et al. concluded that shift work causes an increase in the body weight, which in turn, results in an increase in body mass index (BMI) and waist circumference. Shift workers are exposed to greater health risks compared to those who work standard hours (6). Parkes et al. in their studies showed that for those working at day or night, BMI rises more in comparison with a day working shift works (7). Moreover, shift work has both long-term and short-term effects. Its short-term effects include effects on sleeping, circadian rhythm, performance, and safety as well as disorders in one's individual and social life, while the long-term effects include gastrointestinal problems as well as obesity and cardiovascular diseases (8). Studies have found nutrition of night shift workers to be poorer than that of day workers. This is due to lack of availability of healthy foods for those working at night as well as different meal patterns compared to day workers. Other lifestyle behaviors may also lead to poorer health outcomes among shift workers, for instance in relation to smoking, alcohol consumption and physical activity (9). Also, BMI is a measure of weight adjusted for height, calculated as weight in kilograms divided by the square of height in meters $\left(\mathrm{kg} / \mathrm{m}^{2}\right)(10)$. Unfortunately, shift work can also be very disruptive to a person's health and wellbeing. Shift work is also linked to additional problems with physical and mental health, performance and safety. Shift work is an occupational characteristic of military personnel and this study aimed to assess the effects of shift work on obesity among military personnel in 2016.

\section{Material and Methods}

This descriptive study was carried out on 80 man military personnel at southern Iran, in June-July 2016. The two groups were similar in terms of the type of work. The subjects were selected through simple random sampling. The participants were divided into two groups based on their working schedule as day work (from 7 am to $4 \mathrm{pm}, \mathrm{n}=40$ ) and shift work (from $7 \mathrm{pm}$ to $7 \mathrm{am}, \mathrm{n}=40$ ).

Data collection tools consisted of a tape measure with a precision of $1 \mathrm{~cm}$ and a digital balance (NBL 223e model, Adam Equipment Inc, USA) with a precision of $0.001 \mathrm{~g}$.

Respondents were ensured that their information would remain anonymous and the data would be kept in a safe place and would not be used for any purposes other than for the present study. An approval was also taken from the Ethics Committee. Inclusion criteria were no history of cardiovascular diseases or other medical problems in the file of respondents and 6-month shift work experience.

The BMI is defined as the body mass divided by the square of the body height and is universally expressed in units of $\mathrm{kg} / \mathrm{m}^{2}$, resulting from the mass in kilograms and height in meters. The BMI is an attempt to quantify the amount of tissue mass (muscle, fat, and bone) in an individual. Commonly accepted BMI ranges are underweight: under $18.5 \mathrm{~kg} / \mathrm{m}^{2}$, normal weight: 18.5 to 25 , overweight: 25 to 30 , obese: over 30 (10). In the present study, first, the subjects' height was measured in terms of the meter using a tape measure. Then, their weight was measured in terms of $\mathrm{Kg}$ using a digital balance, and then, BMI was calculated [weight $(\mathrm{kg}) /$ height $(\mathrm{m})^{2}$ ].

Respondents were also asked for the permission to review their ethical issue. Data collector gave a brief introduction to the participants by explaining the aims and 
benefits of the study. Informed written consent was obtained from all participants. Anonymity and confidentiality of data were maintained throughout the study. We utilized the SPSS software (version 20, IBM Corporation, Armonk, NY, USA) to analyze the obtained data using descriptive statistics, analysis of variance (ANOVA) and Pearson correlation tests. Also, the value of $\mathrm{P}<0.05$ was considered statistically significant.
This was a retrospective study that carried out on 80 military personnel (40 shift work and 40 day work), aged from 26 to 39 years, in 2016. Mean age of participants was $35.41 \pm 6.07$ years. Demographic variables of participants including age and work experience are shown in table 1 . According to that, it can be concluded that the average age $(\mathrm{P}=0.03)$ and work experience $(\mathrm{P}=0.02)$ of shift workers were more than day workers.

\section{Results}

Table 1: Demographic variables of the participants $(n=80)$

\begin{tabular}{cccc}
\hline Variable & Type shift & Mean \pm SD & Minimum-maximum \\
\hline \multirow{2}{*}{ Age (year) } & Day work & $33.00 \pm 2.07$ & $26-35$ \\
\cline { 2 - 4 } & Shift work & $38.00 \pm 3.15$ & $27-39$ \\
\hline \multirow{2}{*}{ Work experience (year) } & Day work & $9.07 \pm 1.74$ & $2-11$ \\
\cline { 2 - 4 } & Shift work & $14.17 \pm 2.31$ & $3-16$ \\
\hline
\end{tabular}

SD: Standard deviation

The basic characteristics (height, weight and BMI) of the study population of shift workers and day workers are presented in table 2 .
According to that, mean body weight and BMI in shift work were more than day work individuals.

Table 2: Basic characteristics of the participants $(n=80)$

\begin{tabular}{cccc}
\hline Variable & Type shift & Mean \pm SD & Minimum-maximum \\
\hline \multirow{2}{*}{ Height $(\mathbf{c m})$} & Day work & $183.00 \pm 1.04$ & $170-189$ \\
\cline { 2 - 4 } & Shift work & $182.00 \pm 2.65$ & $171-186$ \\
\hline \multirow{2}{*}{ Weight $(\mathbf{k g})$} & Day work & $78.40 \pm 4.26$ & $71-84$ \\
\cline { 2 - 4 } & Shift work & $86.37 \pm 7.17$ & $75-97$ \\
\hline \multirow{2}{*}{ BMI $\left(\mathbf{K g} / \mathbf{m}^{\mathbf{2}}\right)$} & Day work & $22.18 \pm 1.26$ & $18.9-25.8$ \\
\cline { 2 - 4 } & Shift work & $26.79 \pm 2.87$ & $24.6-32.2$ \\
\hline
\end{tabular}

BMI: Body mass index; SD: Standard deviation

In this study, Kolmogorov-Smirnov test showed that the distribution of variables was normal. Furthermore, findings revealed that mean body weight and BMI in shift workers is more than day workers. The results also showed that $79.6 \%$ of subjects had BMI $>25$ in shift work individuals, with control age and work experience. Also, $83.9 \%$ of subjects had $\mathrm{BMI}<25$ in day work individuals.

ANOVA test showed a significant association between age $(\mathrm{P}=0.01)$ and work experience
$(\mathrm{P}=0.01)$ with $\mathrm{BMI}$ in shift work individuals. Finally, ANOVA test showed a significant association between age $(\mathrm{P}=0.04)$ and work experience $(\mathrm{P}=0.04)$ with $\mathrm{BMI}$ in day work individuals.

\section{Discussion}

Shift work and long work hours increase exposures to hazards at work and deteriorate the health. The purpose of this study was to 
examine the effects of shift work on obesity among military personnel. The immediate effects of shift work can include stress, fatigue, discomfort, and physiological dysfunction. Research indicates that the influence of shift work and long work hours on health and safety may involve a complex interaction of work schedule characteristics (11).

The results of this study showed that the average age and work experience in shift workers are more than day workers. Moreover, the results of this study showed that prevalence of overweight and obesity significantly increased with increasing shift work duration. Ishizaki et al. investigated the impact of the work type on BMI in a factory in Japan and concluded that individuals' shift work, BMI and waist-hip ratio were more than day workers (12). These results have been confirmed in our present study. Lorenzo et al. studied the effect of shift work on BMI and showed that shift work has an effect on weight and BMI (13), consistent with our findings. Antunes Conceição et al. investigated the relationship of shift work and waist circumference, BMI and depressive symptoms and concluded that shift work is a risk factor for obesity and metabolic syndrome (14), which all are in line with our results. The results of this study significantly showed that increased shift work duration is directly associated with enhanced BMI and body weight, consistent with the results of Antunes et al. study (15). Khademian et al. investigated the relationship between night work and nurses anthropometric indices and concluded that shift work has the incremental impact of on BMI (16). Morikawa et al. investigated the effect of shift work on BMI and metabolic parameters. Consistent with our findings, they showed that shift work is considered to be a risk factor for excess weight and there was a significant difference between body weight of shift worker and day worker (17). Weight gain may be induced by long working through reduced sleep duration, decreased physical activity, and undesirable behaviors (18).

It has been suggested that shift work is a factor in nurses' risk for both obesity and unhealthy diet. More research is needed to identify whether the impact is due to the time of day or night worked, length of shift, hours worked, or shift work's impact on sleep quality. Occupational health nurses must be cognizant of the fact that shift work, extended work hours, and poor sleep quality may be risk factors for weight gain for all workers, not only for nurses (19). Gholami Fesharaki et al. investigated the relationship between shift work and obesity and concluded weekly shift workers have lower BMI than day workers. Also, there was no significant statistical difference between BMI of routine shift workers and day workers (1), which is consistent with the findings of the present study. The findings of Ghanbary Sartang research showed that shift work increased the risk of overweight and obesity. The findings of this study showed that $81.6 \%$ of shift workers had a BMI of higher than $25 \mathrm{~kg} / \mathrm{m}^{2}$, also $86.2 \%$ of day workers had a BMI of less than $25 \mathrm{~kg} / \mathrm{m}^{2}$ (20). The relationship between shift work and obesity has been observed in longitudinal studies such as the study by Peplonska et al. their results support the previously reported association between night shift work and development of obesity, which is consistent with the findings of the present study. The findings of Peplonska et al. research showed cumulative night shift work significant association with BMI increasing by $0.477 \mathrm{~kg} / \mathrm{m}^{2}$ per 1000 night duties and by $0.432 \mathrm{~kg} / \mathrm{m}^{2}$ per 10000 night shift hours (21). Previous researchers have identified an effect of shift work on obesity among older age groups. However, there is insufficient information regarding shift work-associated obesity among younger age groups (22). The findings of Garaulet research showed that shift work was related to a higher risk of obesity in individuals, which is consistent with the findings of the present study (23). 
The present study has several limitations. First, the study population does not reflect the general population, because it only included man military personnel. Second, the relatively small sample size of this study highly recommends conducting studies with a bigger population.

\section{Conclusion}

The shift work caused an increased risk of overweight, obesity and BMI and shall be taken to intervention actions in shift works. Interventive actions in shiftwork system similar job rotation can have a major impact on increasing the health of military personnel.

\section{Acknowledgments}

The study was designed and financially supported by the military center. We would like to appreciate all the people involved in this research project, particularly $\mathrm{Mr}$. Ghasemi.

Conflict of interest: None declared.

\section{References}

1. Gholami Fesharaki M, Kazemnejad A, Zayeri F, Rowzati M, Akbari H. Relationship between shift work and obesity a retrospective cohort study. Journal of Military Medicine 2012; 14(2):93-7.

2. Suwazono Y, Dochi M, Sakata K, Okubo Y, Oishi M, Tanaka K, et al. A longitudinal study on the effect of shift work on weight gain in male Japanese workers. Obesity (Silver Spring) 2008; 16(8):1887-93.

3. Macagnan J, Pattussi MP, Canuto R, Henn RL, Fassa AG, Olinto MT. Impact of nightshift work on overweight and abdominal obesity among workers of a poultry processing plant in southern Brazil. Chronobiol Int 2012; 29(3):336-43.

4. Bellanger TM, Bray GA. Obesity related morbidity and mortality. J La Med Soc 2005; 157(1):S42-9.

5. Antunes LC, Levandovski R, Dantas G, Caumo W, Hidalgo MP. Obesity and shift work: chronobiological aspects. Nutr Res Rev 2010; 23(1):155-68.
6. Dorrian J, Skinner N. Alcohol consumption patterns of shift workers compared with dayworkers. Chronobiol Int 2012; 29(5):6108.

7. Parkes KR. Shift work and age as interactive predictors of body mass index among offshore workers. Scand J Work Environ Health 2002; 28(1):64-71.

8. Spengos K, Tsivgoulis G, Manios E, Tsivgoulis A, Zakopoulos N, Vemmos KN. Circadian and seasonal distribution of cardioembolic strokes due to atrial fibrillation. Hellenic J Cardiol 2004; 45(4):234-41.

9. Sakata K, Suwazono Y, Harada H, Okubo Y, Kobayashi E, Nogawa K. The relationship between shift work and the onset of hypertension in male Japanese workers. J Occup Environ Med 2003; 45(9):1002-6.

10. Eknoyan G. Adolphe Quetelet (1796-1874)-the average man and indices of obesity. Nephrol Dial Transplant 2008; 23(1):47-51.

11. Folkard S, Lombardi DA. Modeling the impact of the components of long work hours on injuries and accidents. Am J Ind Med 2006; 49(1):953-63.

12. Ishizaki M, Morikawa $Y$, Nakagawa $H$, Honda R, Kawakami N, Haratani T, et al. The influence of work characteristics on body mass index and waist to hip ratio in Japanese employees. Ind Health 2004; 42(1):41-9.

13. Di Lorenzo L, De Pergola G, Zocchetti C, L'Abbate N, Basso A, Pannacciulli N, et al. Effect of shift work on body mass index: results of a study performed in 319 glucosetolerant men working in a Southern Italian industry. Int J Obes Relat Metab Disord 2003; 27(11):1353-8.

14. Antunes Lda C, Jornada MN, Ramalho L, Hidalgo MP. Correlation of shift work and waist circumference, body mass index, chronotype and depressive symptoms. Arq Bras Endocrinol Metabol 2010; 54(7):652-6.

15. Antunes LC, Levandovski R, Dantas G, Caumo W, Hidalgo MP. Obesity and shift work: chronobiological aspects. Nutr Res Rev 2010; 23(1):155-68.

16. Khademian Z, Saadat F, Hasanshahi $S$. Relationship between night work and Nurses' anthropometric indices. Iran Journal of Nursing 2012; 25(76):77-84.

17. Morikawa Y, Nakagawa H, Miura K, Soyama Y, Ishizaki M, Kido T, et al. Effect of shift work on body mass index and metabolic parameters. Scand J Work Environ Health 2007; 33(1):45-50.

18. Lovejoy JC, Sainsbury A; Stock Conference 2008 Working Group. Sex differences in 
obesity and the regulation of energy homeostasis. Obes Rev 2009; 10(2):154-67.

19. Buss J. Associations between obesity and stress and shift work among nurses. Workplace Health Saf 2012; 60(10):453-8.

20. Ghanbary Sartang A, Ashnagar M, Habibi E, Nowrouzi I, Ghasemi H. The relationship of body mass index and waist-hip ratio with shift work among military personnel in 2016. Journal of Occupational Health and Epidemiology 2015; 4(4):252-9.

21. Peplonska B, Bukowska A, Sobala W.
Association of rotating night shift work with BMI and abdominal obesity among nurses and midwives. PLoS One 2015; 10(7):e0133761.

22. van Amelsvoort LG, Schouten EG, Kok FJ. Impact of one year of shift work on cardiovascular disease risk factors. J Occup Environ Med 2004; 46(7):699-706.

23. Garaulet M, Ordovás JM, Madrid JA. The chronobiology, etiology and pathophysiology of obesity. Int J Obes (Lond) 2010; 34(12):1667-83. 